\title{
Assessment of Linear Switched Reluctance Motor's Design Parameters for Optimal Performance
}

\author{
Jordi Garcia-Amoros ${ }^{1}$, Pere Andrada ${ }^{2}$, and Balduí Blanque ${ }^{2}$ \\ ${ }^{1}$ Electrical and Electronic Engineering Department, University Rovira i Virgili, Tarragona, Spain \\ ${ }^{2}$ GAECE-EPSEVG, Universitat Politècnica de Catalunya, UPC-BarcelonaTech, Vilanova i la Geltrú, Spain
}

\begin{abstract}
This paper presents an exhaustive study about the influence of some design parameters, such as the number of phases, the pole stroke, and the current density, in the behavior of Linear Switched Reluctance Motor (LSRM). LSRM's performance is assessed taking into account a set of quality indices, which are the energy conversion loop quality factor, the propulsion force per unit of primary steel volume, per unit of copper mass, per unit of air gap surface and finally the force ripple factor. The study is carried out by means of the 2-dimensional Finite Element analysis. Finally, the results are discussed and as a consequence a set of LSRM configurations with optimal performance according to the quality indices is defined.
\end{abstract}

Keywords linear switched reluctance machines, finite element analysis, machine design, and sensitivity analysis.

\section{NOMENCLATURE}

$B_{p}$ Magnetic flux density peak in the active pole $(T)$

$b_{p}$ Primary pole width $(m)$

$b_{s}$ Secondary pole width $(m)$

$c_{p}$ Primary slot width $(m)$

$c_{s}$ Secondary slot width $(m)$

$g$ Air gap length $(m)$

hy Primary yoke height $(m)$

$I_{B} \quad$ Flat-topped current peak $(A)$

$J_{B}$ Current density peak $\left(A / \mathrm{mm}^{2}\right)$ 
$K_{S}$ Slot fill factor

$L_{a u}$ Unsaturated aligned inductance $(H)$

$L_{a s}$ Saturated aligned inductance $(H)$

$L_{s}$ Saturated aligned incremental inductance $(H)$

$L u$ Unaligned inductance $(H)$

$l_{p} \quad$ Primary pole length $(m)$

$l_{s} \quad$ Secondary pole length $(m)$

$L_{W}$ Stack length $(m)$

$m$ Number of phases

$N_{l}$ Number of coils per pole

$N_{p p}$ Number of active poles per phase

$N_{s}$ Number of passive poles per side (Secondary)

PS Pole stroke $(m)$

$S$ Distance between aligned and unaligned positions $(\mathrm{m})$

$T_{p}$ Primary pole pitch $(\mathrm{m})$

$T_{s}$ Secondary pole pitch $(m)$

$x \quad$ Mover position $(m)$

\section{INTRODUCTION}

In recent years, Linear Switched Reluctance Motors (LSRMs) and its rotating counterpart (SRM) have been rapidly developed as they have improved their main drawbacks: force ripple and acoustic noise. LSRM have been proposed for a wide range of applications such as force actuator [1], precise motion control [2], for propulsion railway transportation systems [3], active vehicle's suspension system [4], life-support applications [5] and in direct-drive wave energy conversion [6]. LSRMs are an attractive alternative to permanent magnet linear motors (PMLM), despite the fact the force/volume ratio is about 60 per cent lower for LSRMs [7]. In contrast, the absence of permanent magnet makes them less expensive, easy assembling and provides a greater robustness and a good fault tolerance capability.

The LSRM consist of two parts: the active part or primary part, and the passive or secondary. The active part contains the windings and defines two main types of LSRMs: transverse and longitudinal. Longitudinal 
when the plane that contains the flux lines is parallel to the line of movement and transverse when it is perpendicular. Other classification is considering the windings totally concentrated in one coil per phase [2] or partially concentrated in two poles per phase (i.e. single-sided) or four poles per phase (double-sided) $[1,3]$. Figure 1 shows all the possible configurations belonging to this classification. The simplest structure is the single-sided flat LSRM shown in Fig. 1a in which the number of stator active poles is $2 \cdot m$, and the number of poles per phase $\left(N_{p p}\right)$ is 2. A conventional double-sided flat LSRM (see Fig. 1b) is created by joining two single-sided structures, in this case $N_{p p}=4$ and the number of stator active poles is $N_{p}=N_{p p} \cdot m$, where $m$ is the number of phases.

The double-sided structure (figures $1 \mathrm{~b}$ and $1 \mathrm{c}$ ) balances the normal force over the mover and therefore the linear bearing does not have to support it. This configuration has twice air-gaps and coils than singlesided, which means a double propulsion force. Conventional double sided (figure 1b) can operate with one flux loop or two flux loops due to the magnetic connection between secondary poles. In the modified doublesided LSRM (figure 1c) the mover is comprised of rectangular poles without connecting iron yokes between them but are mechanically joined by non-magnetic mounting parts [8]. This arrangement reduces the mass of the mover, giving a higher translation force/mass ratio than conventional double-sided flat LSRM, which reduce the mover weight and its inertia although only allows operating with one flux loop. The tubular structure is shown in figure $1 \mathrm{~d}$.

In previous papers the authors first studied the influence of the flat-LSRM geometry [9], optimizing the poles main dimensions (see figure 2) normalized to the primary pole pitch $\alpha_{p}=b_{p} / T_{p}, \beta_{p}=l_{p} / T_{p}, \alpha_{s}=b_{s} / T_{p}$, $\beta_{s}=l_{s} / T_{p}, \delta_{y}=h_{y} / T_{p}$, regarding to the average propulsion force and concluding that the optimal pole shape values were for: $\alpha_{p}=0.42 ; \beta_{p}=2.5 ; \alpha_{s}=0.5 ; \beta_{s}=0.5 ; \delta_{y}=0.54$. In second place, they proposed a design procedure for this kind of motors [10] and finally they presented a simulation model of a double-sided LSRM acting as a force actuator [1]. This paper completes the research about the double-sided LSRM, giving a comprehensive study of five quality indices: the energy conversion loop quality factor, the specific force per unit primary steel volume $\left(\mathrm{N} / \mathrm{m}^{3}\right)$, the force per unit copper mass $(\mathrm{N} / \mathrm{Kg})$, the force per unit air gap 
surface $\left(\mathrm{N} / \mathrm{m}^{2}\right)$ and the force ripple factor. These indices define the performance indices set named $\mathcal{P}$. The present study is focused in the longitudinal modified double-sided LSRM (see figure 1c and figure 2) and searches the LSRM configurations that optimized these quality indices by means of an exhaustive investigation over the number of phases $(m)$, the pole stroke $(P S)$, the current density $(J)$ and the mover position $(x)$, which define the search space set called $\mathcal{R}$.

The paper is organized as follows: section II presents the model description and the analytical mathematical model, section III presents the formulation of the analysis, section IV discuss the obtained results and finally section $\mathrm{V}$ outlines the conclusions drawn from this research.

\section{ANALYTICAL MODEL DESCRIPTION}

The LSRM's is built from a set of design parameters called $(\mathcal{M})$ which comprises the number of phases $(m)$, the pole stroke $(P S)$, all the magnetic circuit dimensions (see figure 2), such as $b_{p}, l_{p}, b_{s}, l_{s}, h_{y}$ the airgap length $(g)$, the stack width $\left(L_{W}\right)$ and the operating parameters such as the current density $J$, the mover position $x$ and the slot fill factor $\left(K_{s}\right)$. The magnetic circuit dimensions are normalized to the primary pole pitch $T_{p}$, resulting the set of design parameters $\mathcal{M}$ :

$$
\mathcal{M}=\left\{m, P S, J, x, \alpha_{p}, \beta_{p}, \alpha_{s}, \beta_{S}, \delta_{y}, g, L_{W}, K_{S}\right\}
$$

The search space set satisfies: $\mathcal{R} \subset \mathcal{M}$ and results: $\mathcal{R}=\{m, P S, J, x\}$. The analysis $\mathcal{P}(\mathcal{R})$ is carried out by means a 2D-FEM in the modified double-sided structure whose main dimensions are shown in figure 2 .

From (1), the number of poles (2) and the pole pitches (3) are obtained.

$$
\left.\begin{array}{c}
\begin{array}{c}
N_{p}=2 \cdot m \\
N_{S}=2 \cdot(m-1)
\end{array} \\
\begin{array}{c}
T_{p}=(m-1) \cdot P S \\
T_{S}=m \cdot P S
\end{array}
\end{array}\right\}
$$

The pole stroke $P S$ is defined as the distance covered by the mover from an aligned position to the next aligned position when two consecutive phases are excited. The stator length $L$ can be expressed as:

$$
L=(m-1) \cdot\left[(2 \cdot m-1)+\alpha_{p}\right] \cdot P S
$$


The LSRM frame-size is defined as the product $L \cdot L_{W}$ which is also the air gap surface. The numbers of wires per pole $\left(N_{l}\right)$ and the wire's cross section $\left(S_{c}\right)$ are parameters that characterize the phase winding and define the slot fill factor $\left(K_{S}\right)[10]$.

$$
K_{S}=\frac{2 \cdot N_{1} \cdot S_{c}}{\left(1-\alpha_{p}\right) \cdot \beta_{p} \cdot T_{P}^{2}}
$$

The fundamentals of the reluctance machines lay on the energy conversion loop shown in figure 3 . Discarding iron and friction losses, the average thrust force available per phase $\left(F_{X, a v g}\right)$ is then obtained by:

$$
F_{X, a v g}=\frac{2 \cdot \operatorname{area~} 0 A B 0}{m \cdot P S}
$$

In order to simplify the analytical model, the energy conversion loop (see figure 3) is approximated by three straight lines, $0 \mathrm{C}, \mathrm{CB}$ and $0 \mathrm{~A}$ which define the aligned unsaturated inductance $L_{a u}(0 \mathrm{C})$, the aligned saturated inductance $L_{s}(\mathrm{CB})$ and the unaligned inductance $L_{u}(0 \mathrm{~A})$.

The utilization factor $K_{L}$ is defined as a quality factor over the energy conversion loop as:

$$
K_{L}=\frac{\operatorname{area~} 0 A B 0}{I_{B} \cdot \psi_{B}}
$$

This factor is a measure of the energy converted (area $0 \mathrm{AB} 0)$ with regard to the energy available $\left(\Psi_{B}\right.$. $I_{B}$ ). Assuming that area $0 \mathrm{AB} 0 \approx$ area $0 \mathrm{ABC} 0$ and $L_{s}=L_{u}$, then $K_{L}$ results:

$$
K_{L}=\left(1-\frac{L_{u}}{L_{a s}}\right) \cdot\left(1-\frac{1}{2} \cdot \frac{L_{a s}-L_{u}}{L_{a u}-L_{u}}\right)
$$

Rearranging (6), results [9]:

$$
F_{X, a v g}=4 \cdot \frac{m-1}{m} \cdot K_{L} \cdot K_{S} \cdot \alpha_{P} \cdot\left(1-\alpha_{P}\right) \cdot \beta_{P} \cdot L_{W} \cdot T_{P}^{2} \cdot\left(B_{P} \cdot J_{B}\right)
$$

The average shear force $\left(\mathrm{N} / \mathrm{m}^{2}\right)$ is defined as:

$$
f_{X S, a v g}=\frac{F_{X, a v g}}{L \cdot L_{W}}=4 \cdot \frac{(m-1) \cdot K_{L} \cdot K_{S} \cdot \alpha_{P} \cdot\left(1-\alpha_{P}\right) \cdot \beta_{P}}{m \cdot(2 \cdot m-1) \cdot \alpha_{P}} \cdot\left(B_{P} \cdot J_{B}\right)
$$

And the average specific force $(N / \mathrm{kg})$ is defined as:

$$
f_{X C u, a v g}=\frac{F_{X, a v g}}{M_{C u}}=4 \cdot\left(\frac{m-1}{m \cdot \gamma_{C u}}\right) \cdot \frac{K_{L} \cdot \alpha_{P} \cdot L_{W} \cdot B_{P} \cdot J_{B}}{4 \cdot L_{W}+\pi \cdot T_{P}^{2} \cdot\left(1-\alpha_{P}\right)}
$$

Where $M_{C u}=V_{C u} \cdot \gamma_{C u}$ is the copper mass per phase, $V_{C u}$ is the copper volume per phase and $\gamma_{\mathrm{Cu}}$ is its specific mass $\left(8890 \mathrm{~kg} / \mathrm{m}^{3}\right.$ for copper). Finally, the average density force is defined as: 


$$
f_{X V p p, a v g}=\frac{F_{X, a v g}}{V_{p p}}=\frac{m-1}{m^{2}} \cdot K_{L} \cdot K_{S} \cdot\left(1-\alpha_{P}\right) \cdot\left(B_{P} \cdot J_{B}\right)
$$

Where $V_{p p}$ is the primary steel pole volume: $V_{p p}=4 \cdot m \cdot \alpha_{p} \cdot \beta_{p} \cdot T_{p}^{2} \cdot L_{W}$. It is also possible to define the average density force taking the whole primary steel volume $\left(V_{p}\right)$, which is: $V_{p}=V_{p p}+2 \cdot \delta_{y} \cdot T_{p} \cdot L_{W} \cdot L$, being:

$$
V_{p}=\left[4 m \alpha_{p} \beta_{p}+2 \delta_{y}\left(2 m+\alpha_{p}-1\right)\right] L_{W}(m-1)^{2} P S^{2}
$$

\section{FORMULATION OF THE ANALYSIS}

In order to focus the study, the parameters which define the magnetic circuit dimensions $\left\{\alpha_{p}, \alpha_{s}, \beta_{p}, \beta_{s}\right.$, $\left.\delta_{y}\right\}$ are assigned for optimizing the poles shape [9] $\left(\alpha_{p}=0.42 ; \alpha_{s}=0.5 ; \beta_{p}=2.5 ; \beta_{s}=0.5 ; \delta_{y}=0.54\right)$. The air gap length is set to $g=0.5 \mathrm{~mm}$ according to the mechanical tolerances. The stack width $\left(L_{W}\right)$ is proportional to the propulsion force $\left(F_{X} \propto L_{W}\right)$ since 2D FEM doesn't account for end-effects, and therefore, this parameter acts as a scale factor for the force. It is adjusted according to a required propulsion force, $L_{W}=30$ $\mathrm{mm}$ in this case. The slot fill factor is fixed to 0.5. Finally, taking into account all the previous considerations, the set of model design parameters results: $\mathcal{M}=$ $\{m, P S, J, x, 0.5,2.5,0.5,0.54,0.5,30,0.5\}$, where the search space is $\mathcal{R}=\{m, P S, J, x$,$\} , in which the$ searching ranges are $m \in\{2,3,4,5\}, P S \in\{3,4,5,6,7,8,9,10 \mathrm{~mm}\}$ and $J \in[0.5,20], \Delta J=0.5 \mathrm{~A} / \mathrm{mm}^{2}$. For each combination of $\{m, P S, J\}$ there are 21 positions $(x)$ evenly distributed between the pole misalignment $(x=0)$ and the pole alignment $(x=S)$, where $S$ is the distance between the aligned and the unaligned positions given by $S=m \cdot P S / 2, x \in[0, S]$. The total number of computed problems is $21 \times 8 \times 4 \times 40 \times(2+3+4+5)=235200$. The LSRM's geometry is automatically generated for each pair $\{m, P S\} \subset \mathcal{M}$. Figure 4 shows several LSRM geometry examples. The steel grade used is a standard M 270-50A.

The study is carried out by taking the set of LSRM performance indices $\mathcal{P}$, defined as:

$$
\mathcal{P}=\left\{K_{L}, f_{X V p, a v g}, f_{X C u, a v g}, f_{X S, a v g}, f_{\text {ripple }}\right\}
$$

The analysis of $\mathcal{P}(\mathcal{R})$ is performed using a 2D-FEM solver [11]. The thrust or propulsion force $F_{X}(15)$ is calculated by the Maxwell stress tensor over a surface enclosing the whole mover. In 2D FEM, this surface is determined by the path $\Gamma$ multiplied by the depth $L_{W}$. Thus for a given point of $\mathcal{R}$ : 


$$
F_{X}(m, P S, J, x)=\frac{L_{W}}{\mu_{0}} \cdot \oint_{\Gamma} n_{y} \cdot B_{x} \cdot B_{y} \cdot d l
$$

In general the $j$-phase linked flux $\Psi_{j}$ can be computed integrating $\vec{B}$ over the inner surface $S_{i}$ of each one of the $N_{l}$ wires and for each one of the $N_{p p}$ poles per phase.

$$
\Psi_{j}=\sum_{k=1}^{N_{p p}} \sum_{i=1}^{N_{1}} \int_{S_{i}} \bar{B}_{i k} \cdot d \bar{S}_{i}
$$

In $2 \mathrm{D}$, due to the impossibility to take into account the end-winding, equation 16 is rearranged in terms of the line integral of the vector potential $(\vec{A})$ over the wire's side path of length $L_{W}$ (see figure 2). The difference of the line integral of $\vec{A}$ over both coil sides is the linked flux. Assuming the winding cross section as $S_{c u+}$, the sign (+) denotes where the current flow is positive, and $S_{c u-}$ where the current flows in opposite direction, the vector potential can be averaged over these areas, thus for a given point of $\mathcal{R}$, the whole $j$ phase winding flux linkage is obtained from:

$$
\Psi_{j}(m, P S, J, x)=N_{p p} \cdot N_{1} \cdot L_{W} \cdot\left(\frac{1}{S_{C u^{+}}} \iint_{S_{c u^{+}}} A_{j} \cdot d S-\frac{1}{S_{C u^{-}}} \iint_{S_{c u^{-}}} A_{j} \cdot d S\right)
$$

The utilization factor $K_{L}$ is computed from (7), where the area OABO (see figure 3) is obtained integrating (17). The density force $f_{X V p p}\left(N / \mathrm{m}^{3}\right)$ is defined in (18) as the force per stator steel volume $V_{p}(13)$. The force per unit copper mass $f_{X C u}\left(N / \mathrm{m}^{3}\right)$ is defined in (19) and the shear force $f_{X S}\left(\mathrm{~N} / \mathrm{m}^{2}\right)$ is defined in (20).

$$
\begin{gathered}
f_{X V p}(x, m, P S, J)=\frac{F_{x}(x, m, P S, J)}{V_{p}(m, P S)} \\
f_{X C u}(x, m, P S, J)=\frac{F_{x}(x, m, P S, J)}{M_{C u}(m, P S)} \\
f_{X S}(x, m, P S, J)=\frac{F_{x}(x, m, P S, J)}{L_{W} \cdot L(m, P S)}
\end{gathered}
$$

The forces $f_{X V p p}, f_{X C u}$ and $f_{X S}$ are averaged (21-23) in order to eliminate the position dependence.

$$
\begin{gathered}
f_{X V p, a v e}(m, P S, J)=\frac{1}{S} \int_{0}^{S} f_{X V p}(x, m, P S, J) d x \\
f_{X C u, a v e}(m, P S, J)=\frac{1}{S} \int_{0}^{S} f_{X C u}(x, m, P S, J) d x \\
f_{X S, a v e}(m, P S, J)=\frac{1}{S} \int_{0}^{S} f_{X S}(x, m, P S, J) d x
\end{gathered}
$$


The force ripple has been one of the main drawbacks of reluctance devices. Up to date, force ripple has been treated by modifying the geometry poles [9, 12, 13], implementing a current control strategy [14], by finding the optimal firing position [1] and by seeking for the optimal spatial arrangement of a multimodular LSRM [15]. The ripple factor is defined in (24) for a flat current waveform illustrated in figure 5, where the phase conduction interval for each $\operatorname{LSRM}(m, P S)$ is $P S$.

$$
f_{\text {ripple }}(m, P S, J)=\frac{f_{x, \max }-f_{x, \min }}{f_{x, \text { ave }}}
$$

Table 1 collects all the performance indices computed, their units and their variable dependencies.

\section{RESULTS AND DISCUSSION}

Figure 6 depicts the plots of $K_{L}(m, P S, J)$ for $J \in\{5,10,15,20\} \mathrm{A} / \mathrm{mm}^{2}$. As it is expected, $K_{L}$ decreases as $J$ increases, the absolute maximums are reached at $K_{L}(4,10,5), K_{L}(3,10,10), K_{L}(3,8,15)$ and $K_{L}(3,7,20)$.

The average density force $\left(f_{X V p, a v e}\right)$ is a measure of the use of the steel lamination and it is shown the whole set $\{m, P S, J\}$ in figure 7. The wrapping curve, which contains the highest values, presents the same tendency as $K_{L}$, that is, for low $J$ and $P S$ the relative maximum is revealed at higher $m$. The absolute maximum is reached at $m=3$ phases in all the cases.

Figure 8 shows the force per unit copper mass $\left(f_{X C u, a v e}\right)$, which can be seen as a measure of the use of copper mass. These results show the absolute maximums organized in descending order for $m$. For high current density $\left(J=20 \mathrm{~A} / \mathrm{mm}^{2}\right)$ the rule changes and $m=4$ phases slightly exceeds $m=5$ phases.

Figure 9 presents the average shear force results. The curves follow the same pattern as the previous cases. Figure 10 shows the $f_{\text {ripple. }}$. As it is expected, the ripple factor decreases as the number of phases increases. Nevertheless it is interesting to notice that for $m=4$ phases, $P S=4 \mathrm{~mm}$ and $J=10 \mathrm{~A} / \mathrm{mm}^{2}$ the ripple factor reach a minimum, being even lower than for $m=5$ phases. The same singular behavior is observed for $J=5$ $\mathrm{A} / \mathrm{mm}^{2}, m=4$ phases and PS=5 $\mathrm{mm}$ and $6 \mathrm{~mm}$.

In order to summarize the study results, figure 11 collects the $\{m, P S, J\}$ configurations which have an absolute maximum in the performance indices set $\mathcal{P}$. 
For $J=5 \mathrm{~A} / \mathrm{mm}^{2}$, which can be a suitable value for operating in a continuous duty cycle $\mathrm{S} 1$, there are two premium configurations which are optimal in all the performance indices at $m=5$ phases and $P S=3 \mathrm{~mm}$ and $4 \mathrm{~mm}$. For higher pole stroke there are several high-performance configurations with 2 or 3 optimal average force indices, that is, in $m=5$ phases and $P S=5 \mathrm{~mm}$, in $m=4$ phases $(P S=6 \div 8 \mathrm{~mm})$ and in $m=3$ phases $(P S=9$ $\mathrm{mm}$ and $10 \mathrm{~mm})$.

For $J=10 \mathrm{~A} / \mathrm{mm}^{2}$ (continuous duty cycle S1 with an improved cooling system, intermittent periodic duty $\mathrm{S} 3$ or continuous operation periodic duty cycle S6), the premium performance is given at $m=5$ phases $P S=3 \mathrm{~mm}$. The high-performance configurations are: at $m=5$ phases $(P S=4 \mathrm{~mm})$, at $m=4$ phases $(P S=5 \mathrm{~mm}$ and $6 \mathrm{~mm})$, at $m=3$ phases $(P S=7 \div 10 \mathrm{~mm})$.

For higher current densities, $J=15$ and $20 \mathrm{~A} / \mathrm{mm}^{2}$ (LSRM operating in short time duty S2 or intermittent periodic duty S3 with forced cooling), there are not premium configurations. For $J=15 \mathrm{~A} / \mathrm{mm}^{2}$ the highperformance configurations are: at $m=5$ phases $(P S=3 \mathrm{~mm})$, at $m=4$ phases $(P S=4 \mathrm{~mm}$ and $5 \mathrm{~mm})$, at $m=3$ phases $(P S=6 \div 10 \mathrm{~mm})$. For $J=20 \mathrm{~A} / \mathrm{mm}^{2}$ the high-performance configurations are: at $m=4$ phases $(P S=3$ $\mathrm{mm}$ and $4 \mathrm{~mm})$, at $m=3$ phases $(P S=5 \div 8 \mathrm{~mm})$, at $m=2$ phases $(P S=9 \mathrm{~mm}$ and $10 \mathrm{~mm})$.

Table 2 gives the length of the LSRM's optimal configurations which at least optimize the use of the steel $\left(f_{X V p, a v g}\right)$ and copper $\left(f_{X C u, a v g}\right)$. The highlighted in light grey optimize the three average forces and the premium are in dark grey.

The relatively large lengths and moderated current density of the premium configurations (see table 2) predicts a good cooling condition and therefore their viability is assured. For $m=4$ phases only two of the high-performance configurations ( $P S=7 \mathrm{~mm}$ and $8 \mathrm{~mm}$ ) are prone to avoid overheating due their large size and low current density. For $m=3$ phases the configurations more feasible are for $P S \geq 6 \mathrm{~mm}$ with moderate current density $J \leq 10 \mathrm{~A} / \mathrm{mm}^{2}$. For $m=2$ phases the optimal are for $J=20 \mathrm{~A} / \mathrm{mm}^{2}$ and due to its reduced size these configurations are going to be overheated and therefore are unfeasible.

In order to verify the analysis, the simulated $\operatorname{LSRM}(4,4)$ force propulsion results are contrasted with an existing LSRM $(4,4)$ prototype [10], whose geometrical parameters, in parenthesis, are slightly different from the simulated case, $\alpha_{p}=0.42(0.5) ; \alpha_{s}=0.5(0.583) ; \beta_{p}=2.5(2.5) ; \beta_{s}=0.5(0.583) ; \delta_{y}=0.54(0.666)$. The 
simulated 2D FEM force has to be corrected for the end-effects, which is done by means of an estimating procedure which defines the end-effect correction factor $K_{e e}[9,10]$. The prototype slot fill factor $\left(K_{S, p}=0.423\right)$ is slightly lower respect to the LSRM simulated $\left(K_{S}=0.5\right)$, thus the simulated force accounting for the end-effects and the slot fill factor variation is obtained from:

$$
F_{X, 3 D}(m, P S, J)=\left(\frac{K_{S, p}}{K_{S}}\right) \cdot K_{e e} \cdot F_{X}(m, P S, J)
$$

Figure 12 shows the comparison results between $F_{X, 3 D}(4,4)$ and the prototype force measurements. These results show a good agreement for the range of current densities measured $\left(J=5,10,15\right.$ and $\left.20 \mathrm{~A} / \mathrm{mm}^{2}\right)$, which validates the presented analysis.

\section{CONCLUSION}

This paper has presented a novel and detailed study about the influence of number of phases $m$, pole stroke $P S$ and current density $J$ over the performance of LSRM according to a set of performance indices. This study has revealed an optimal set of LSRM configurations, which is useful information for LSRM designers and can summarize as:

1) The best configuration is for $m=5$ phases and $P S=3 \mathrm{~mm}$, which optimizes all the performance indices for $J=5$ and $10 \mathrm{~A} / \mathrm{mm}^{2}$. The configuration $m=5$ phases and $P S=4 \mathrm{~mm}$, optimizes all the performance indices for $J=5 \mathrm{~A} / \mathrm{mm}^{2}$.

2) $m=4$ phases shows a high-performance at $P S=4 \mathrm{~mm}$, but for $J=15$ and $20 \mathrm{~A} / \mathrm{mm}^{2}$ a forced cooling system is required. For $P S=6 \div 8 \mathrm{~mm}$ the high-performance is at $J=10 \mathrm{~A} / \mathrm{mm}^{2}$, which guarantees their viability.

3) $m=3$ phases shows a high-performance at $P S=7 \div 10 \mathrm{~mm}$ for $J=10$ and $15 \mathrm{~A} / \mathrm{mm}^{2}$ which are feasible configurations, intermittent periodic duty S3 or continuous operation periodic duty cycle S6.

4) $m=2$ phases shows a high-performance at high current density and small sizes which predicts overheating. The ripple factor is two times higher than for $m=3$ which means high level of vibration and noise. 


\section{REFERENCES}

[1] Amorós, J.G.; Blanque, B.; Andrada P. "Modelling and Simulation of a Linear Switched Reluctance Force Actuator". IET Electric Power Applications, vol. 7, Issue 5, pp. 350-359, May 2013

[2] Zhao S. W., Cheung, N.C., Wai-Chuen Gan; Jin Ming Yang; Jian Fei Pan, "A Self-Tuning Regulator for the High-Precision Position Control of a Linear Switched Reluctance Motor”, IEEE Transactions on Industrial Electronics, vol.54, no.5, pp.2425-2434, Oct. 2007

[3] Lobo N. S, Hong Sun Lim, Krishnan R., "Comparison of Linear Switched Reluctance Machines for Vertical Propulsion Application: Analysis, Design, and Experimental Correlation”, IEEE Transactions on Industry Applications. vol.44, no. 4, pp. 1134-1142, Aug 2008

[4] Zhu Zhang; Cheung, N.C.; Cheng, K.W.E.; Xiangdang Xue; Jiongkang Lin; , "Direct Instantaneous Force Control With Improved Efficiency for Four-Quadrant Operation of Linear Switched Reluctance Actuator in Active Suspension System,", IEEE Transactions on Vehicular Technology, vol.61, no.4, pp.1567-1576, May 2012.

[5] Llibre, J.-F.; Martinez, N.; Nogarede, B.; Leprince, P.; "Linear tubular switched reluctance motor for heart assistance circulatory: Analytical and finite element modeling”, 10th International Workshop on Electronics, Control, Measurement and Signals (ECMS), 2011 vol., no., pp.1-6, 1-3 June 2011.

[6] Du, Jinhua; Liang, Deliang; Xu, Longya; et al., "Modeling of a Linear Switched Reluctance Machine and Drive for Wave Energy Conversion Using Matrix and Tensor Approach”, IEEE Transactions on Magnetics, Vol. 46, No. 6, pp 1334-1337, June 2010.

[7] Bianchi, N.; Bolognani, S.; Corda, J. "Tubular linear motors: a comparison of brushless PM and SR motors", International Conference on Power Electronics, Machines and Drives, 2002. (Conf. Publ. No. 487), vol., no., pp. 626- 631, 4-7 June 2002.

[8] Andrada, P.; Blanque, B.; Martínez, E.; Torrent, M.; Amorós, J.G.; Perat, J.I. "New linear hybrid reluctance actuator". Accepted for publication at XXI ICEM, Berlin 2014.

[9] Amoros, J. G.; Andrada, P., "Sensitivity Analysis of Geometrical Parameters on a Double-Sided Linear Switched Reluctance Motor", IEEE Transactions on Industrial Electronics, vol.57, no.1, pp.311-319, January. 2010.

[10] Amorós, J.G.; Andrada, P.; Blanqué, B., "Design Procedure for a Longitudinal Flux Flat Linear Switched Reluctance Motor”, Electric Power Components and Systems, 40:2, 161-178. (2011).

[11] Finite Element Method Magnetics, Vers. 4.2. April 2012. http://www.femm.info 
[12] Sahin, F.; Ertan, H.B.; Leblebicioglu, Kemal, "Optimum geometry for torque ripple minimization of switched reluctance motors", IEEE Transactions on Energy Conversion, vol.15, no.1, pp.30-39, March 2000

[13] Guangjin Li; Ojeda, J.; Hlioui, S.; Hoang, E.; Lecrivain, M.; Gabsi, M., "Modification in Rotor Pole Geometry of Mutually Coupled Switched Reluctance Machine for Torque Ripple Mitigating” IEEE Transactions on Magnetics, vol.48, no.6, pp.2025-2034, June 2012

[14] Pan, J. F.; Cheung, N.C.; Yu Zou, "An Improved Force Distribution Function for Linear Switched Reluctance Motor on Force Ripple Minimization With Nonlinear Inductance Modeling," IEEE Transactions on Magnetics, vol.48, no.11, pp.3064-3067, Nov. 2012

[15] Xiangdang Xue; Cheng, K.E.; Zhu Zhang; Jiongkang Lin; Cheung, N., “A Novel Method to Minimize Force Ripple of Multimodular Linear Switched Reluctance Actuators/Motors", IEEE Transactions on Magnetics , vol.48, no.11, pp.3859-3862, Nov. 2012 


\section{FIGURES}

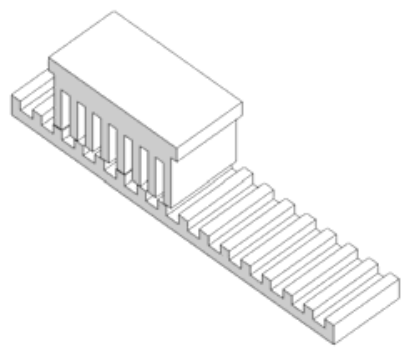

a. Single-sided

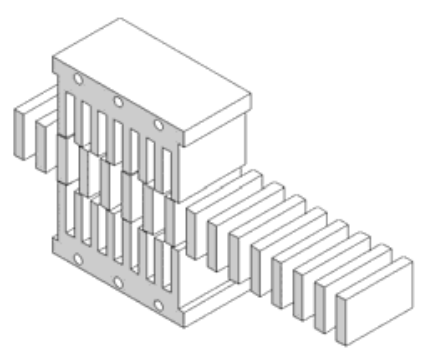

c. Modified double-sided

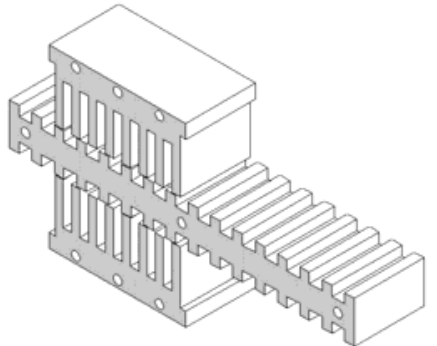

b. Conventional double-sided

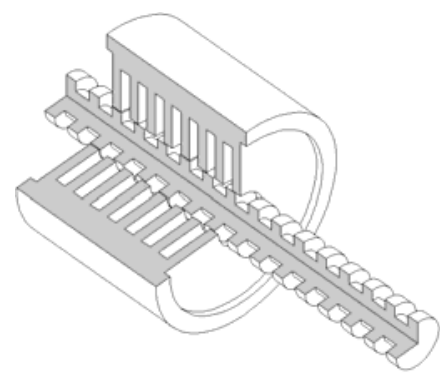

d. Tubular

Fig. 1. Longitudinal flux LSRM topologies.

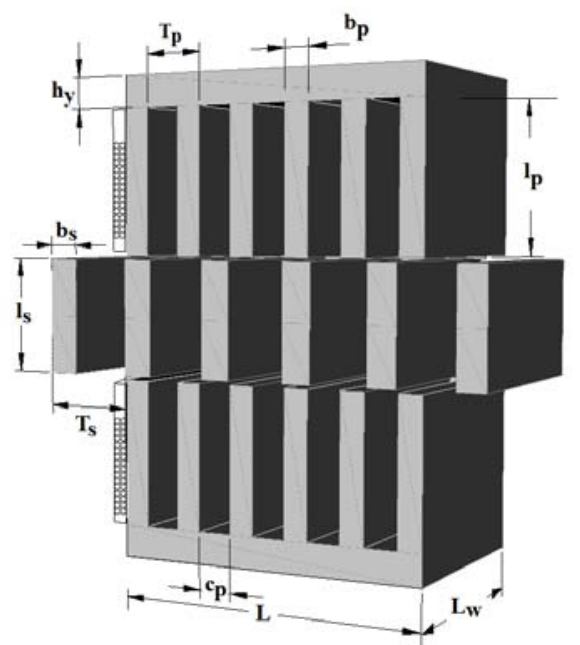

Fig. 2. Modified double-sided LSRM model design parameters (e.g., $m=3$ ). 


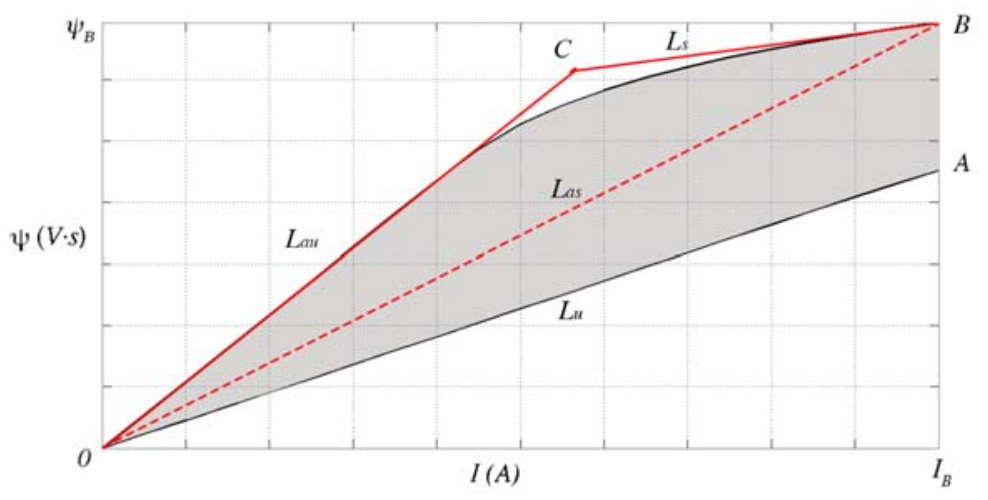

Fig. 3. Energy conversion loop

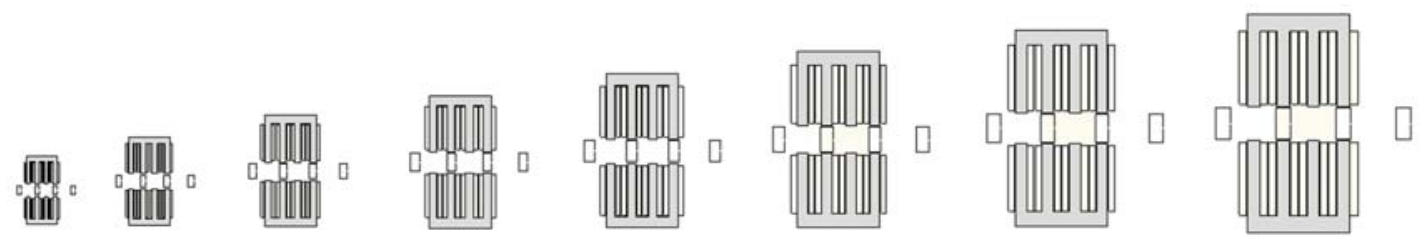

(a) Geometries as function of $P S \in\{3,4,5,6,7,8,9,10 \mathrm{~mm}\}$ for $m=2$.

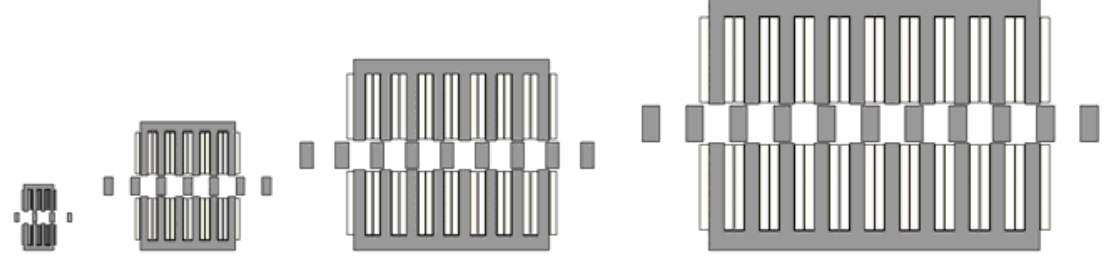

(b) Geometries as function of the number of phases, $m \in\{2,3,4,5\}$ for $P S=3 \mathrm{~mm}$.

Fig. 4. LSRM geometry examples

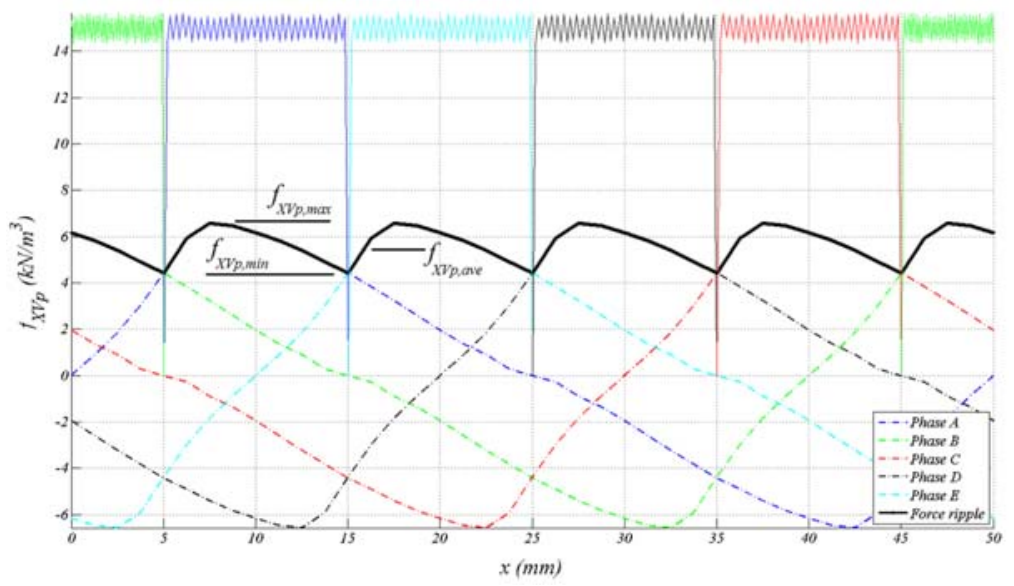

Fig. 5. Force ripple definition (e.g., $\operatorname{LSRM}(5,10))$ 

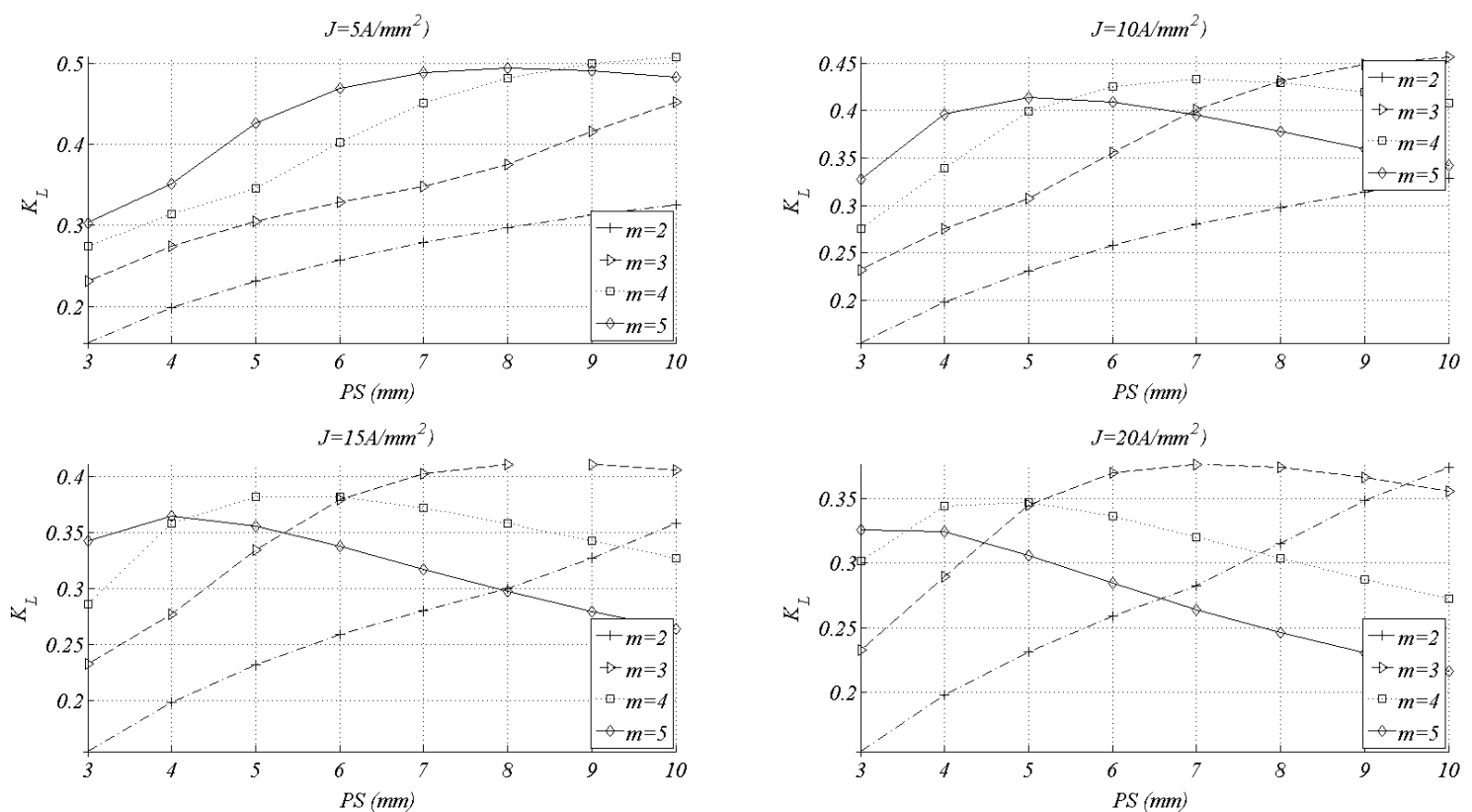

Fig. 6. Utilization factor $K_{L}$.
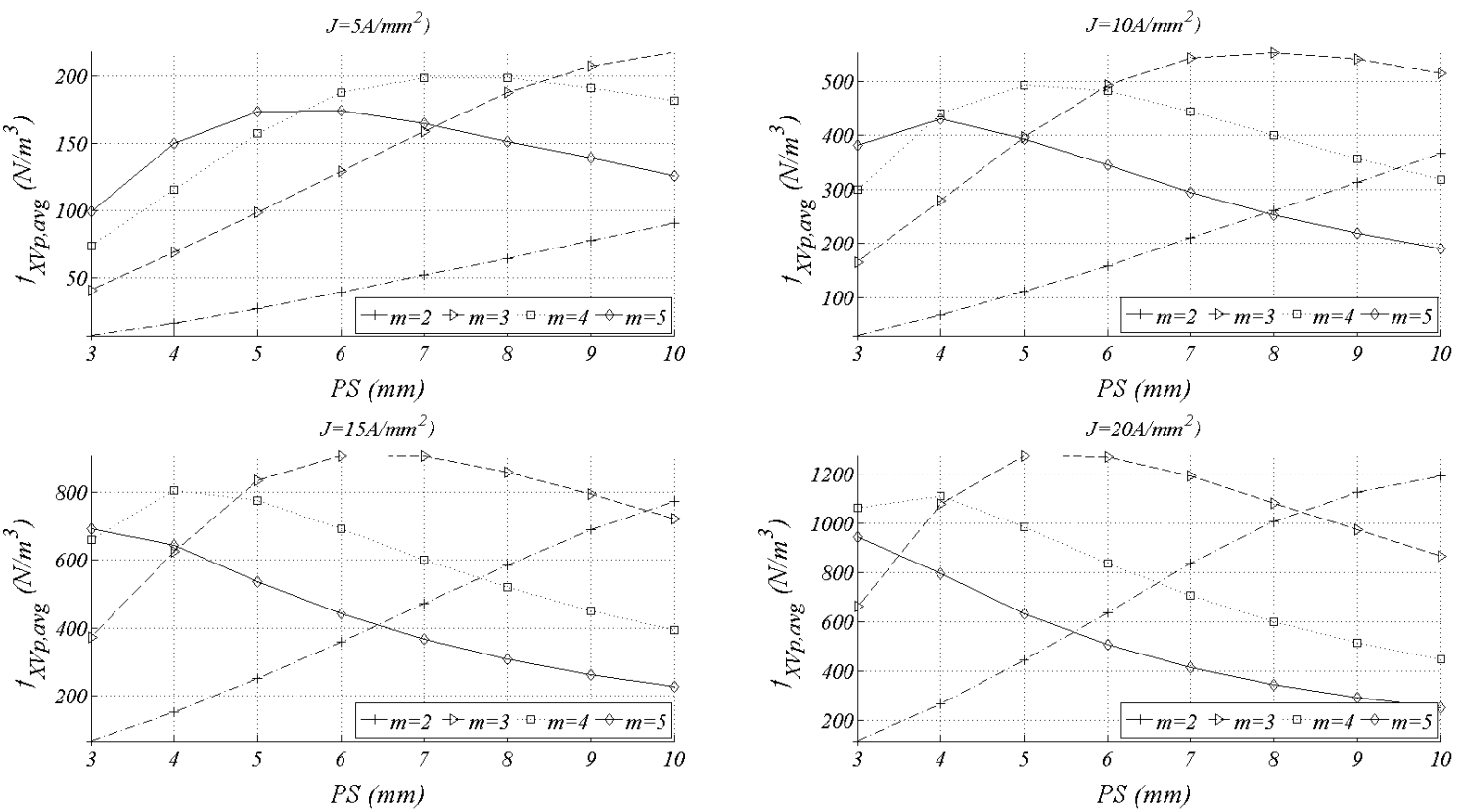

Fig. 7. Average density force results $\left(f_{X V p, a v e}\right)$ 

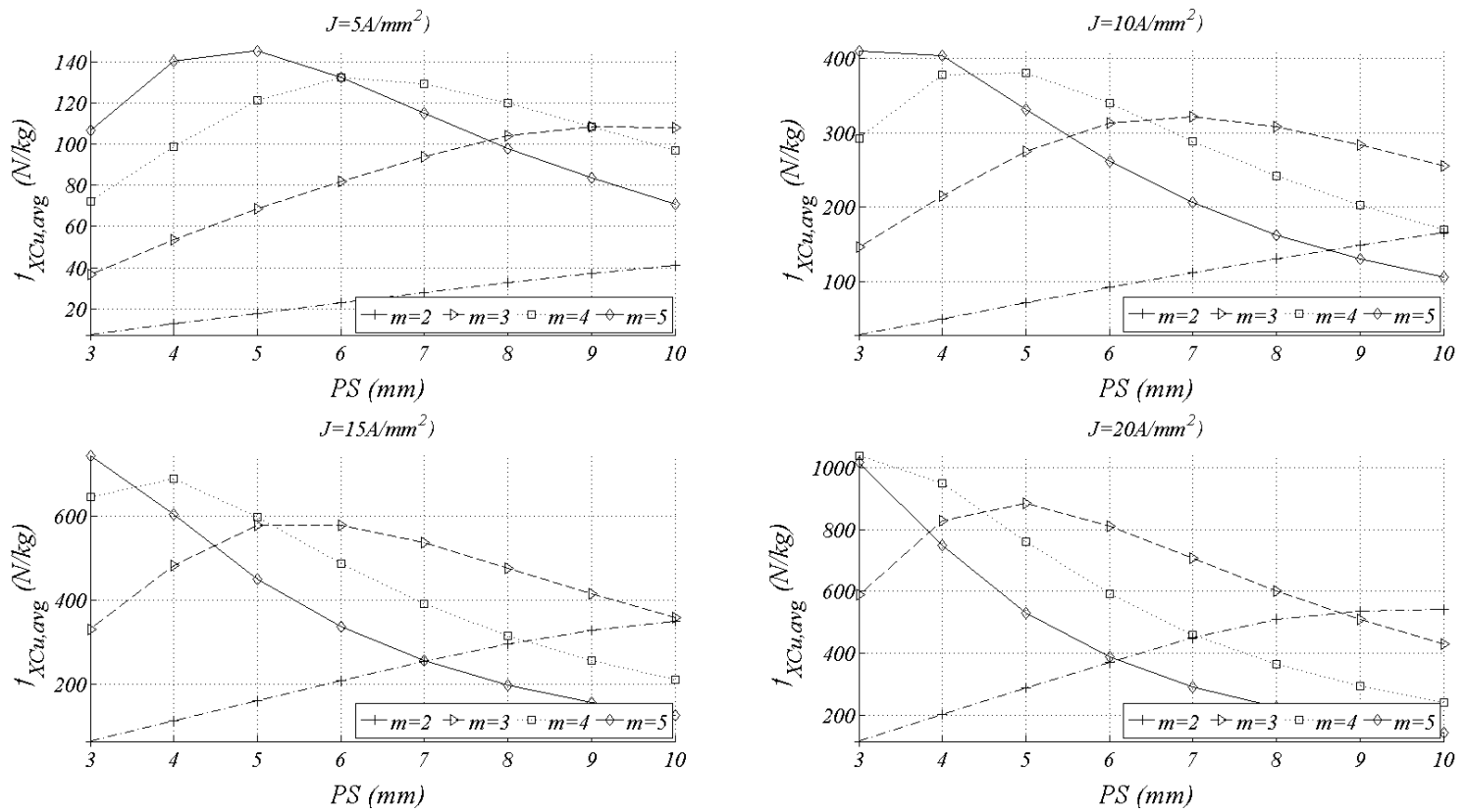

Fig. 8. Average force per unit copper mass $\left(f_{X C u, a v e}\right)$
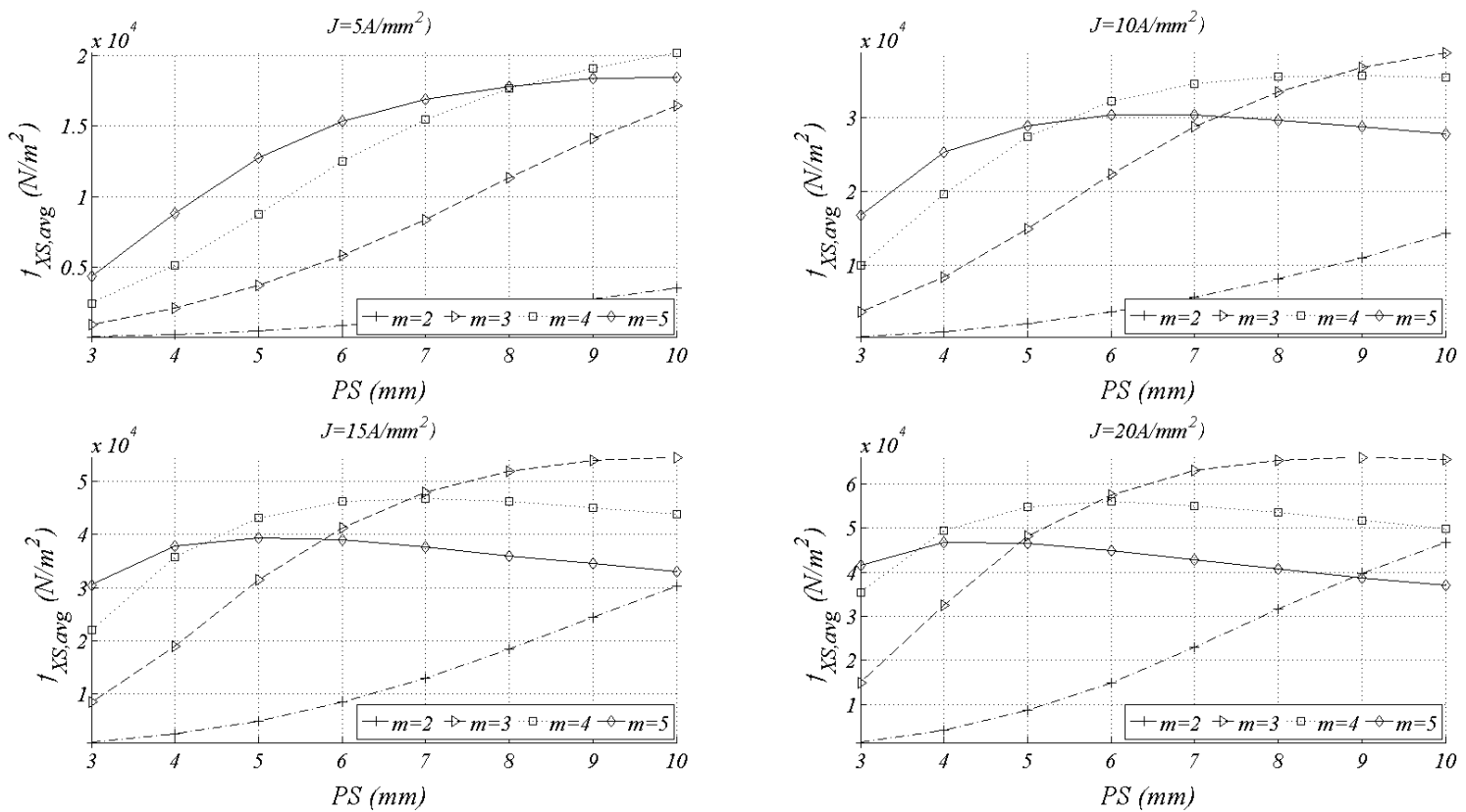

Fig. 9. Average shear force $\left(f_{X S, \text { ave }}\right)$ 

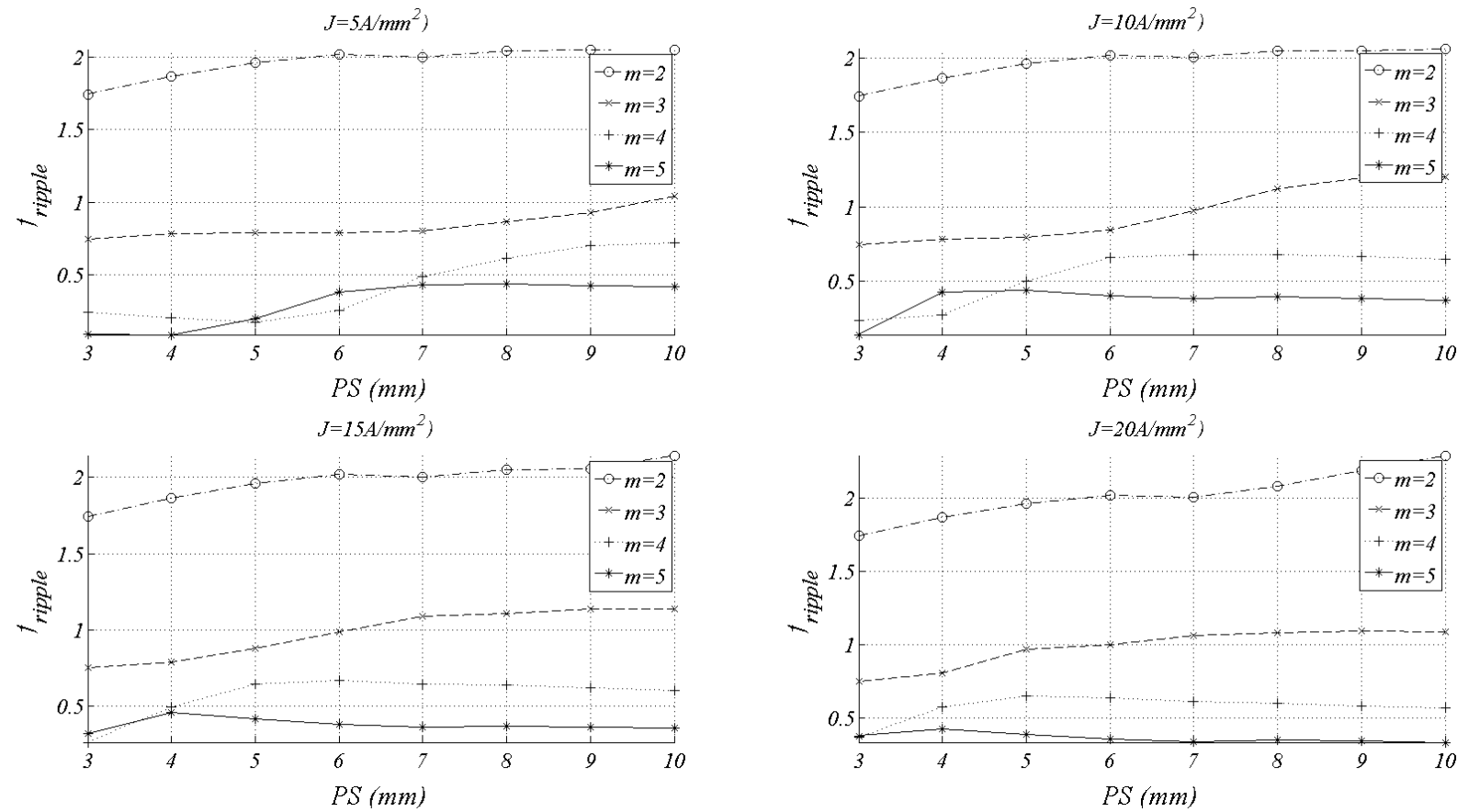

Fig. 10. Ripple factor results
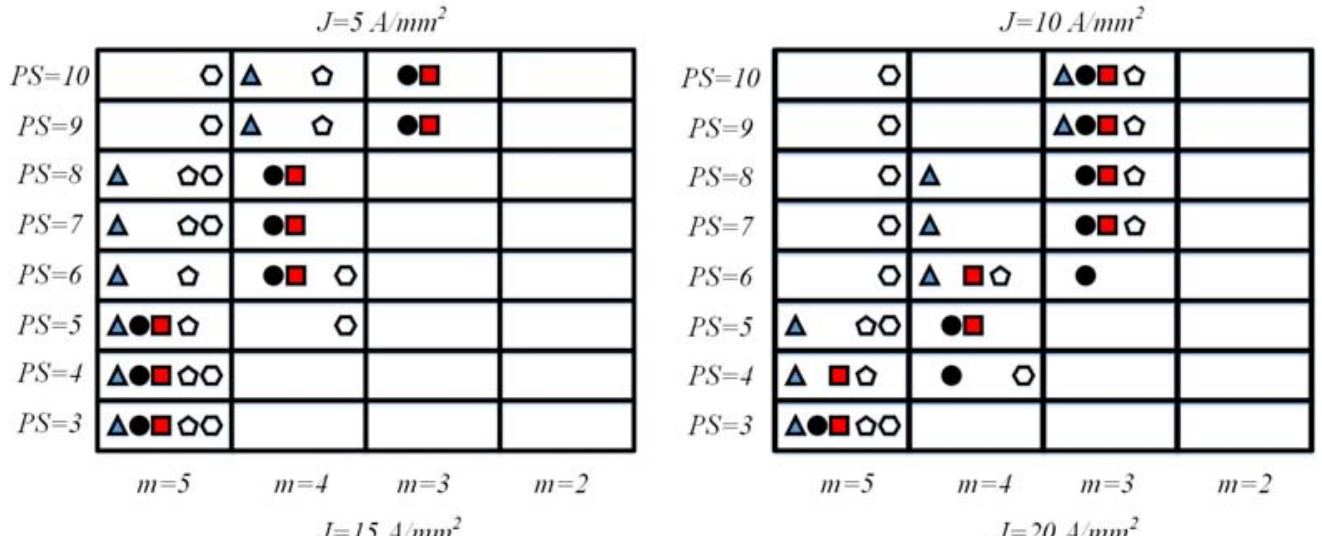

\begin{tabular}{|c|c|c|c|c|}
\hline$P S=10$ & 0 & & $\Delta \square$ & $\bullet$ \\
\hline$P S=9$ & 0 & & $\Delta$ प्व & \\
\hline$P S=8$ & 0 & & $\Delta 0 \square \Delta$ & \\
\hline$P S=7$ & 0 & & $\Delta$ प्व & \\
\hline$P S=6$ & 0 & $\Delta \quad 0$ & 由ा & \\
\hline$P S=5$ & 0 & $\Delta$ प0 & $\bullet$ & \\
\hline$P S=4$ & $\Delta \quad \Delta$ & 由ा & & \\
\hline$P S=3$ & $\Delta 0 \square 0$ & 0 & & \\
\hline
\end{tabular}

\begin{tabular}{|c|c|c|c|c|}
\hline$P S=10$ & 0 & & $\Delta$ & 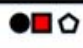 \\
\hline$P S=9$ & 0 & & $\Delta \quad 0$ & ๑ם \\
\hline$P S=8$ & 0 & & $\Delta \bullet \square$ & \\
\hline$P S=7$ & 0 & & $\Delta 0 \square \Delta$ & \\
\hline$P S=6$ & 0 & & $\Delta 0 \square 0$ & \\
\hline$P S=5$ & 0 & $\Delta \quad 0$ & Oa & \\
\hline$P S=4$ & 0 & $\Delta 000$ & & \\
\hline$P S=3$ & $\Delta \quad \Delta$ & 임 & & \\
\hline
\end{tabular}
$\triangle f_{\text {XS, arg }}$
- $f_{X V p, a v g}$
( $f_{\text {XCu,arg }}$
$\checkmark K_{L}$
$\square f_{\text {ripple }}$

Fig. 11. Optimal performance indices configuration 


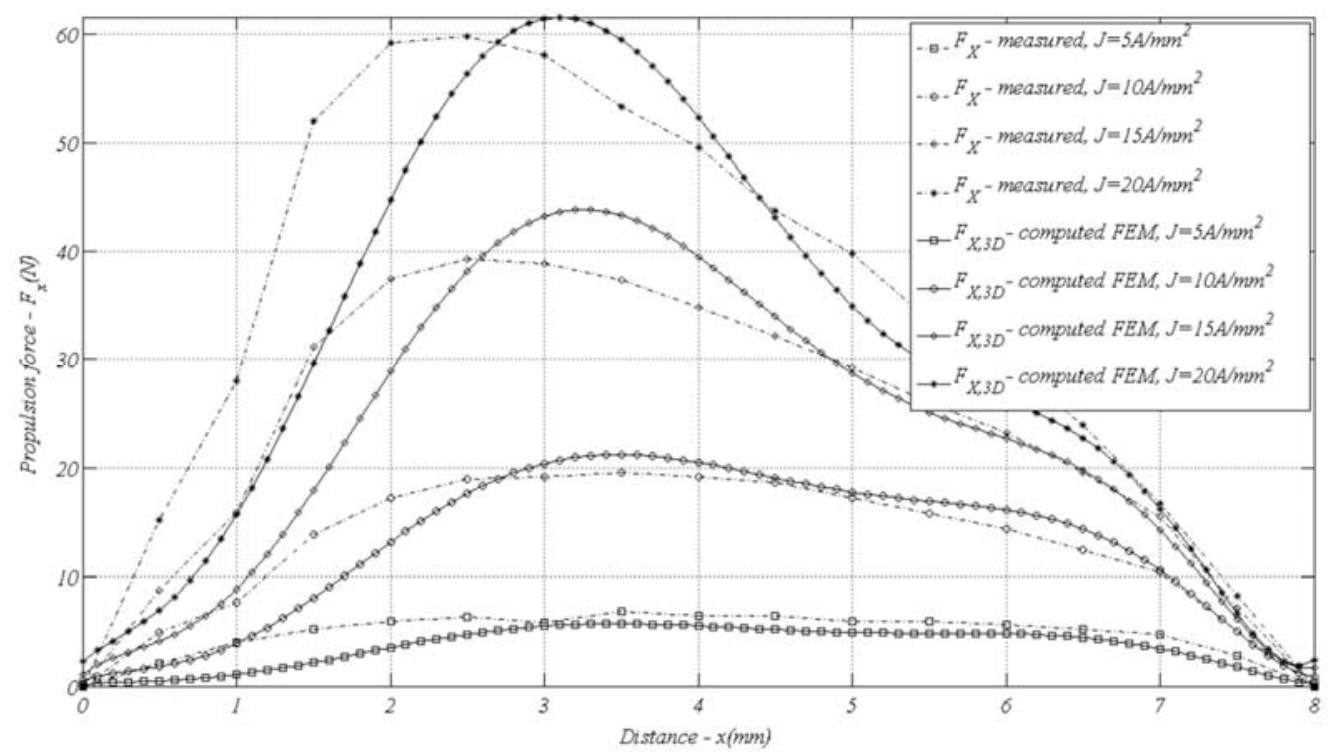

Fig. 12. LSRM $(4,4)$ propulsion force simulated/experimental comparison results

\section{TABLES}

Table 1. Performance indices

\begin{tabular}{|c|c|c|c|c|c|c|c|}
\hline$f_{X V p}$ & $f_{X C u}$ & $f_{X S}$ & $f_{X V p, \text { avg }}$ & $f_{X C u, \text { avg }}$ & $f_{X S, \text { avg }}$ & $f_{\text {ripple }}$ & $K_{L}$ \\
\hline$N / m^{3}$ & $N / k g$ & $N / m^{2}$ & $N / m^{3}$ & $N / k g$ & $N / m^{2}$ & -- & -- \\
\hline \multicolumn{7}{|c|}{$(m, P S, J)$} \\
\hline
\end{tabular}

Table 2. Length (mm) of LSRM optimal configurations

\begin{tabular}{|c|c|c|c|c|c|c|c|c|c|}
\hline \multirow{2}{*}{\multicolumn{2}{|c|}{$\begin{array}{c}L \\
(\mathrm{~mm})\end{array}$}} & \multicolumn{8}{|c|}{$P S(\mathrm{~mm})$} \\
\hline & & 3 & 4 & 5 & 6 & 7 & 8 & 9 & 10 \\
\hline \multirow{4}{*}{ 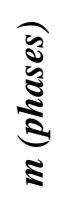 } & & & & & & & & 30,78 & 34,2 \\
\hline & 3 & & & 54,2 & 65,04 & 75,88 & 86,72 & $\mathbf{9 7 , 5 6}$ & 108,4 \\
\hline & 4 & 66,78 & 89,04 & 111,3 & 133,56 & 155,82 & 178,08 & & \\
\hline & 5 & 113,04 & 150,72 & 188.4 & & & & & \\
\hline
\end{tabular}

